\title{
The Use of Fetal Bronchoscopy in the Diagnosis and Management of a Suspected Obstructive Lung Mass
}

\author{
Andrew H. Chon, $\mathrm{MD}^{1}$ James E. Stein, MD, MSc ${ }^{2}$ \\ Walter D. Vazquez, $\mathrm{MD}^{5}$ Ramen H. Chmait, $\mathrm{MD}^{1}$ \\ ${ }^{1}$ Division of Maternal-Fetal Medicine, Department of Obstetrics and \\ Gynecology, Keck School of Medicine, University of Southern \\ California, Los Angeles, California \\ 2 Department of Pediatric Surgery, Children's Hospital Los Angeles, \\ Keck School of Medicine, University of Southern California, \\ Los Angeles, California \\ 3 Division of Maternal-Fetal Medicine, Department of Obstetrics and \\ Gynecology, Kaiser Permanente San Diego, San Diego, California \\ ${ }^{4}$ Department of Pathology and Laboratory Medicine, Keck School of \\ Medicine, University of Southern California, Children's Hospital \\ Los Angeles, Los Angeles, California \\ ${ }^{5}$ Department of General Surgery, Kaiser Permanente San Diego, \\ San Diego, California
}

Am J Perinatol Rep 2018;8:e195-e200.
Tammy Gerstenfeld, DO 3 Larry Wang, MD, $\mathrm{PhD}^{4}$

Address for correspondence Ramen $\mathrm{H}$. Chmait, MD, Division of Maternal-Fetal Medicine, Department of Obstetrics and Gynecology, Keck School of Medicine, University of Southern California, 39 Congress Street, Suite 302, Pasadena, California 91105 LA (e-mail: chmait@usc.edu).

\begin{abstract}
Keywords

- CPAM

- congenital lung mass

- fetal bronchoscopy

Etiologies of fetal lung anomalies include congenital pulmonary airway malformation (CPAM), intra- or extralobar pulmonary sequestration, congenital high airway obstruction syndrome (CHAOS), bronchogenic cyst, and bronchial atresia. Fetal tracheobronchoscopy has been reported both as a diagnostic and therapeutic procedure in the setting of severe congenital lung lesions. In this case report, prenatal imaging of a fetus with a large chest mass was suspicious for an obstructive bronchial lesion. The absence of visible normal lung tissue on the right side and mass effect on the left side raised the concern for pulmonary hypoplasia. After antenatal betamethasone and a period observation, hydropic changes developed. Fetal tracheobronchoscopy was then performed in an effort to identify and decompress the suspected obstructive bronchial lesion. Other than release of bronchial debris, no anatomical abnormalities were visualized. However, the right lung lesion and mediastinal shift both decreased after the fetal bronchoscopy. The newborn underwent postnatal resection of a CPAM Type II and is doing well. We hypothesize that fetal tracheobronchoscopy provided the following potential diagnostic and therapeutic benefits: (1) exclusion of an obstructive bronchial lesion; (2) disimpaction of bronchial debris from the saline lavage that we posit may have contributed to the rapid reduction in CPAM size.
\end{abstract}

A 32-year-old G2P1001 at 24 ${ }^{4 / 7}$ weeks was referred to our center with a large fetal chest mass to possibly undergo fetal therapy. A course of betamethasone was administered to the mother at $23^{0 / 7}$ weeks in the hopes to reduce the size of the lung lesion. ${ }^{1-3}$ Consultation ultrasound revealed a singleton fetus with an estimated fetal weight (EFW) of 810 grams

received

September 11, 2017

accepted

August 21, 2018
DOI https://doi.org/

10.1055/s-0038-1673378. ISSN 2157-6998.
( $78^{\text {th }}$ percentile). Anatomical findings were significant for a large right sided hyperechogenic, homogenous lung that measured $7.3 \times 4.6 \times 3.7 \mathrm{~cm}^{3}$; the $3 \mathrm{D}$ (three-dimensional) volume measured $53.1 \mathrm{~cm}^{3}$. No normal lung tissue was seen on the right side. The CPAM volume ratio (CVR) was 2.7. Arterial systemic feeding vessels to the right lung mass were
Copyright $@ 2018$ by Thieme Medical Publishers, Inc., 333 Seventh Avenue, New York, NY 10001, USA. Tel: +1(212) 584-4662.
License terms

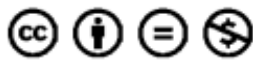




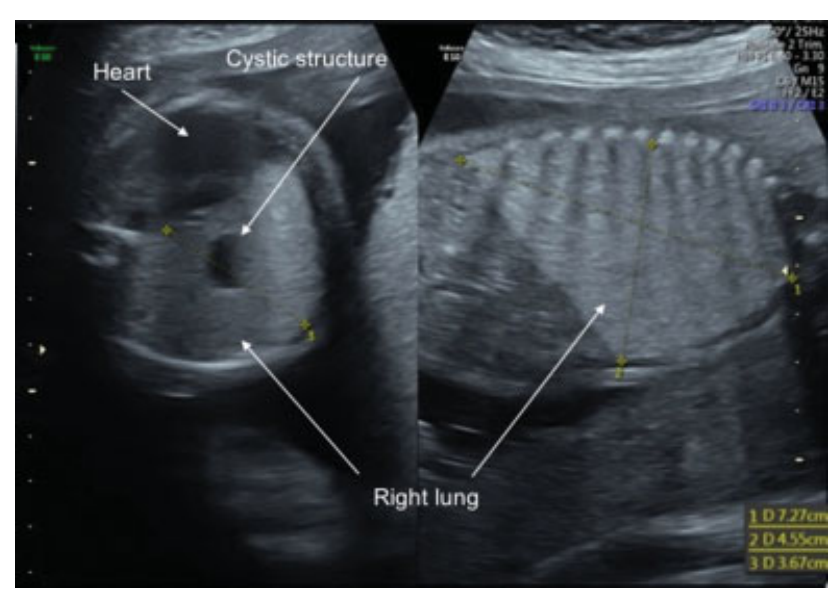

Fig. 1 Ultrasound images from consultation at $24^{4 / 7}$ weeks. (Left) Axial section of the hyperechogenic right lung lesion causing mediastinal shift. (Right) Sagittal section of lung lesion resulting in diaphragmatic flattening.

not visualized. Within the middle of the lung lesion near the area of the right bronchus was a $1.1 \mathrm{~cm}$ diameter hypoechogenic cyst ( - Fig. 1). The left lung was compressed with a quantitative lung index (QLI) of $0.5\left(<0.1^{\text {st }}\right.$ percentile) ${ }^{4}$ There was mediastinal shift and diaphragm eversion present. A small left pleural effusion $(5 \mathrm{~mm}$ ) was noted but there was no other evidence of fetal hydrops at this time. Amniotic fluid maximum vertical pocket (MVP) was $6.2 \mathrm{~cm}$. Thus on ultrasound, the right lung appeared enlarged, hyperechogenic, and homogenous with a solitary cyst near the area of the right bronchus, and the left lung appeared hypoplastic secondary to compression from the mass effect. The differential diagnosis favored bronchial atresia with a bronchocele or an obstructive bronchogenic cyst; however, CPAM and congenital lobar emphysema remained under consideration.

A fetal echocardiogram was performed and the right pulmonary artery was dilated and enlarged pulmonary vessels were seen coursing through the right lung mass in a normal pattern. The cardiac anatomy was otherwise normal. No systemic arterial blood supply to the lung mass was identified. A fetal magnetic resonance imaging (MRI) confirmed the presence of an enlarged right lung with a $\mathrm{T} 2$ hyperintense lesion at the right hilum $\left(1.0 \times 1.3 \times 1.2 \mathrm{~cm}^{3}\right)$, favoring an obstructive bronchogenic cyst or a mucus filled atretic segmental bronchus (-Fig. 2).

The patient was counseled that the risk of pulmonary hypoplasia was high, as there appeared to be minimal normal appearing lung tissue, and that the prognosis remained guarded. Management options were discussed in detail. Expectant management would allow for an opportunity for spontaneous resolution of the lung mass, but more likely the lesion would remain and result in pulmonary hypoplasia. Percutaneous fetal sclerotherapy was not recommended because the primary differential diagnosis favored bronchogenic cyst or right bronchial atresia, and not CPAM Type III. ${ }^{5}$ We offer fetal percutaneous sclerotherapy as a first line treatment to patients with hydrops fetalis secondary to type II or III CPAM. ${ }^{5}$ Direct fetal tracheobronchoscopy was also discussed as an option to evaluate for a bronchial obstruction and if present, perforate with laser energy to allow efflux of pulmonary secretions and restore physiologic pulmonary development, as described by Martinez et al. ${ }^{6}$ The risks of operative fetoscopy including preterm premature rupture of membranes (PPROM) and periviable delivery were discussed. The patient elected expectant management
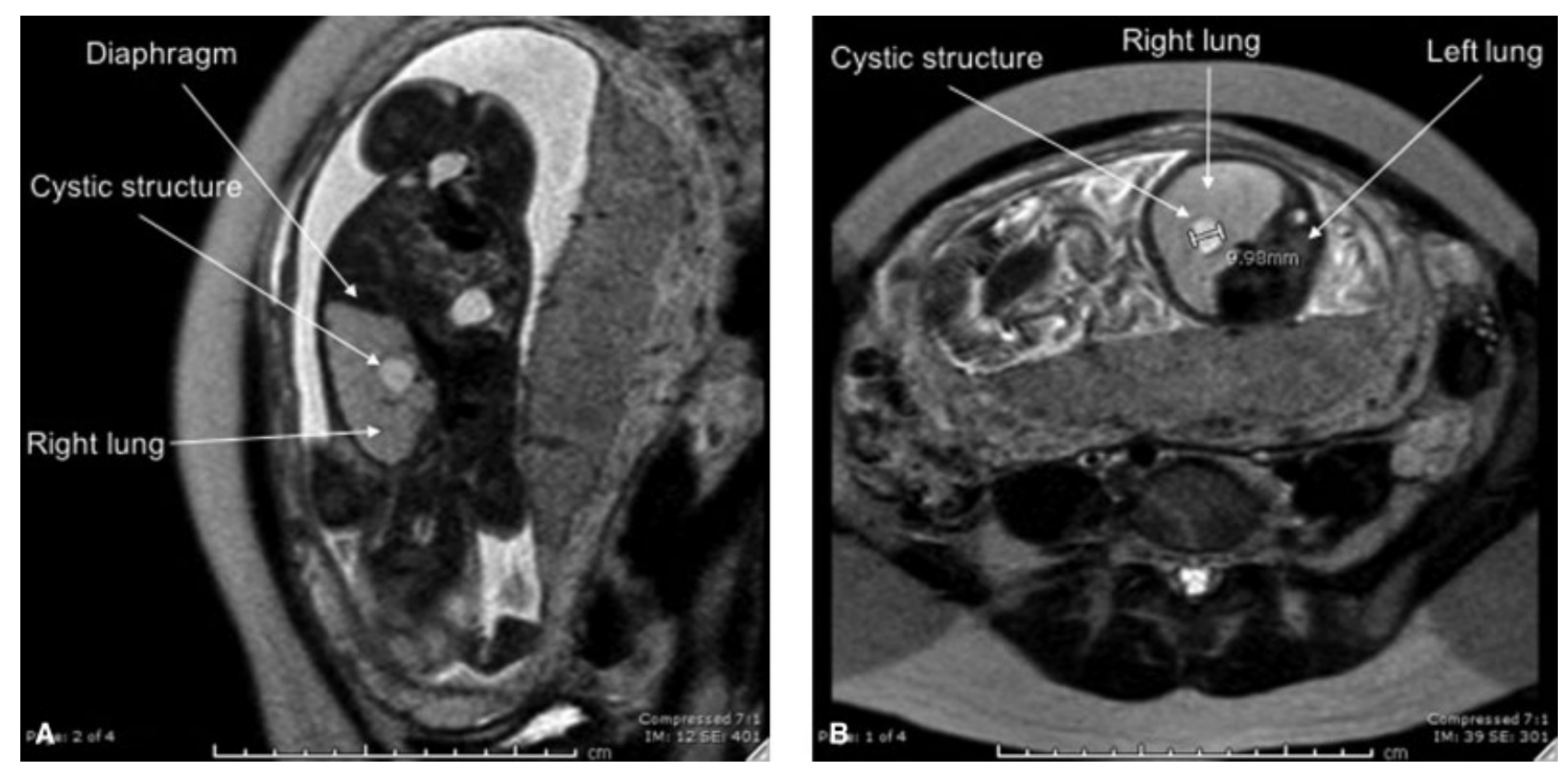

Fig. 2 Fetal T2-weighted MRI at $25^{0 / 7}$ weeks. (A) Sagittal section of the fetus in cephalic position. The enlarged right lung is seen causing diaphragmatic flattening. (B) Axial section of the fetus with the hyperintense cystic structure within the right lung lesion. The left lung is compressed secondary to the mass-effect from the enlarged right lung. 
with the intention of undergoing fetal bronchoscopy if there appeared to be worsening of the fetal condition.

The patient returned for a repeat ultrasound at $25^{6 / 7}$ weeks. The lung findings were unchanged but new onset symptomatic polyhydramnios with a MVP of $9.2 \mathrm{~cm}$ was noted. Given the persistence of the severe lung findings including pleural effusions and new onset polyhydramnios, the patient desired to proceed with an attempt of fetal tracheobronchoscopy. A multidisciplinary meeting was held with Maternal-Fetal Medicine, Neonatology, Pediatric Surgery, and Radiology, and the group agreed that fetal intervention was justified. The stated goal of the fetal tracheobronchoscopy was to decompress the obstructive lesion in the hopes this would resolve the developing hydrops and allow for improved development of the fetal lungs.

A second course of betamethasone was given for the purposes of fetal lung maturation with the added possible benefit of decreasing the size of the lung mass. ${ }^{7,8}$ After obtaining verbal and written consent, the patient was taken to the operating room at $29^{5 / 7}$ weeks. Amniocentesis was performed for fetal karyotype and microarray. Then the needle was redirected and intramuscular injection of fentanyl $(1 \mu \mathrm{g} / \mathrm{kg})$ and rocuronium $(1.5 \mathrm{mg} / \mathrm{kg})$ for fetal anesthesia was provided. Next, operative fetoscopy and fetal tracheobronchoscopy was performed as previously described. ${ }^{9}$ Under ultrasound guidance, a $3.8 \mathrm{~mm}$ cannula with a trocar (Richard Wolf, IL) was inserted into the amniotic cavity. The trocar was removed and a $3.5 \mathrm{~mm}$ 30-degree rigid diagnostic endoscope (Richard Wolf, IL) was used to identify and gain access into the fetal mouth. The cannula was advanced into the fetal mouth and the glottis was identified. The diagnostic endoscope was advanced past the vocal cords and into the trachea where the carina was visualized. The rigid endoscope was advanced into the right and left main bronchus, and both appeared normal. The cannula was held in place above the carina and the rigid endoscope was exchanged for a flexible 4.9 French ureteroscope. Fetal bronchoscopy was then performed to assess the left and right bronchi. Mucoid debris was noted within the bronchi. To improve visualization, intermittent bronchial lavage was performed using normal saline infusion through the operating channel of the ureteroscope. The total volume of saline lavage was estimated at
$60 \mathrm{~mL}$. Both right and left bronchial trees were inspected in all lobes down to the segmental level. All bronchi were patent and had normal branching patterns, ruling out a possible segmental bronchial atresia. Combined direct visualization and ultrasound demonstrated that the $1.1 \mathrm{~cm}$ cystic lesion was not in close proximity (more than $1 \mathrm{~cm}$ away) from the right main or lobar bronchi.

Postoperative ultrasound 24 hours later showed the right lung lesion with a slightly decreased CVR of 2.0. The patient was discharged and followed by her referring perinatologist with serial ultrasounds. The fetal karyotype and prenatal microarray were found to be normal. At $30^{5 / 7}$ weeks the right lung mass had significantly decreased to approximately one-third the size, measuring $4.8 \times 2.5 \times 3.0 \mathrm{~cm}^{3}$, with a calculated volume of $18.7 \mathrm{~cm}^{3}$. At $34^{0 / 7}$ weeks, repeat fetal echocardiogram was notable for improved mediastinal shift and mild cardiomegaly. The last ultrasound at $34^{4 / 7}$ weeks prior to delivery showed the right lung mass measuring $3.3 \times 2.4 \times 3.7 \mathrm{~cm}^{3}$ and calculated volume of $15.2 \mathrm{~cm}^{3}$. The cystic structure within the lung remained stable in appearance. The patient went into labor and underwent a vaginal delivery at $35^{3 / 7}$ weeks.

In the neonatal intensive care unit, the baby was initially supported by continuous positive airway pressure. On day of life 4, computerized tomography (CT) of the thorax showed that the right upper lobe was enlarged, demonstrated air trapping, and also had a unilocular intrapulmonary cyst $\left(1.8 \times 1.2 \times 1.3 \mathrm{~cm}^{3} ;\right.$ - Fig. 3$)$. Due to persistent respiratory insufficiency requiring high-flow nasal cannula, on day of life 9 , the newborn underwent a right thoracotomy and right upper lobectomy. The cyst seen on prior imaging was grossly visualized on the inferior aspect of the right upper lobe. The small size of the cyst prevented the surgeon from determining if it had a clear bronchial connection. Aside from asymptomatic tachypnea, the baby had an otherwise uneventful course and was discharged from the hospital on day of life 26.

At 4 months of age, an echocardiogram was normal. At 6 months of age, the baby required a 5-day hospitalization for an episode of metapneumovirus bronchiolitis. At 8 months of age, due to the persistent tachypnea, the baby underwent a bronchoscopy without abnormal findings. Flexible laryngoscopy was also performed at 9 months of age and demonstrated mild laryngomalacia but normal subglottic lumen
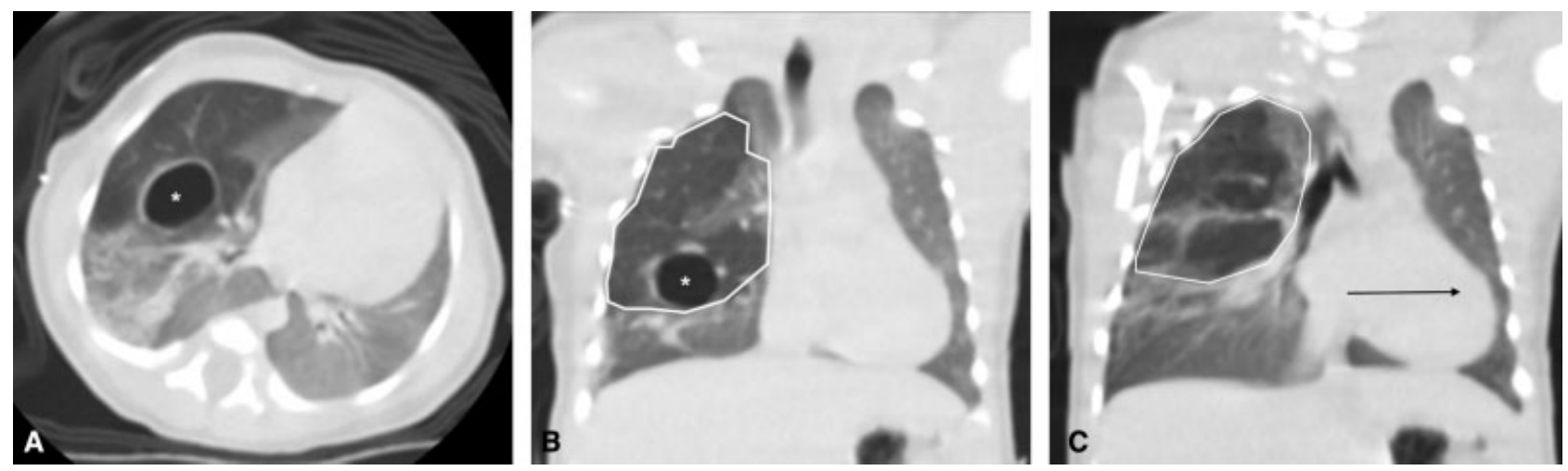

Fig. 3 Computerized tomography scan on day of life 4. (A) Axial view with an intrapulmonary cyst $\left(1.8 \times 1.2 \times 1.3 \mathrm{~cm}^{3}\right)\left({ }^{*}\right)$ in right upper lobe. (B) Coronal view with cyst $\left({ }^{*}\right)$ and prominent lucency suggesting air trapping (outline). (C) Coronal view with secondary effects of hyperinflated right upper lobe (outline) included compressed right middle lobe and right to left mediastinal shift (arrow). 

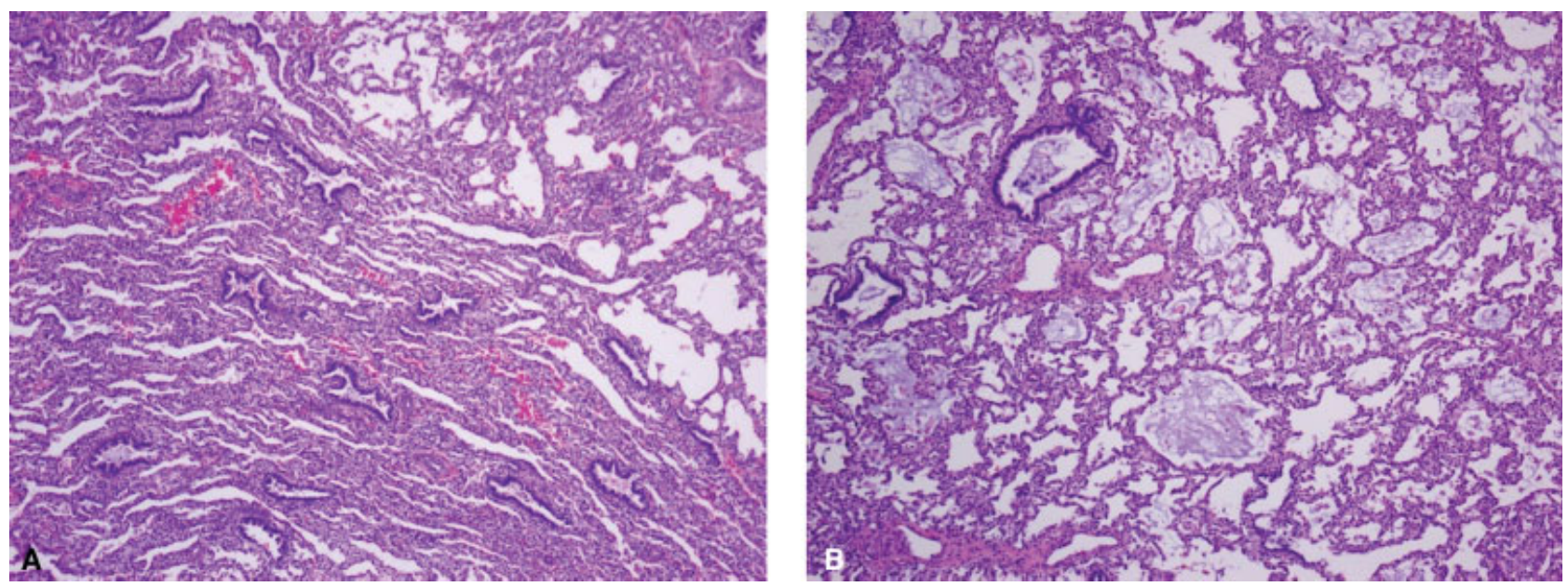

Fig. 4 (A) Hematoxylin and eosin section (magnification: $\times 40$ ) shows several small uniform cysts resembling dilated terminal bronchioles within the lung parenchyma. (B) Section (magnification: $\times 40$ ) shows mucus plugs mixed with few mononuclear cells present within cysts and alveolar spaces.

and normal range of motion of the vocal folds. Aside from bouts of tachypnea, the baby was doing well at 12 months of age without cardiopulmonary issues. The child does not require supplemental oxygen. The baby has pectus excavatum which is being expectantly managed.

Surgical pathology demonstrated a $5.4 \times 4.6 \times 2.0 \mathrm{~cm}^{3}$ (18 grams) specimen with scattered dilated bronchiolar like cysts $(0.5-2.0 \mathrm{~cm})$ as well as possible collapsed cysts up to $1.0 \mathrm{~cm}$ in diameter, consistent with a CPAM Type II ( - Fig. 4). The cysts were lined with a single layer of ciliated columnar respiratory epithelial cells. The cysts were separated by alveolar structures, small arterioles, and venules. The surrounding stroma contained cells with skeletal muscle differentiation. Mucin producing cells were identified. Patchy areas of interstitial emphysema and intra-alveolar mucin inspissation was noted, which resulted in mucus plugs within the cysts and alveolar spaces (-Fig. 4).

\section{Discussion}

In this case report, fetal ultrasound and MRI revealed a large chest mass that was suspicious for an obstructive bronchial lesion. Fetal tracheobronchoscopy provided the following potential diagnostic and therapeutic benefits: (1) exclusion of an obstructive bronchial lesion; (2) disimpaction of bronchial debris from the saline lavage, which we posit may have been a contributing factor to the rapid reduction in CPAM size post procedure.

Differentiating congenital lung lesions prenatally with ultrasound can be challenging as they have several overlapping features. All of these lung lesions have the potential to cause mediastinal shift, diaphragmatic eversion, fetal hydrops, and lethal pulmonary hypoplasia. The finding of a congenital lung lesion requires postnatal histologic followup to ensure an accurate diagnosis. ${ }^{10}$ Furthermore, congenital lung lesions often contain histologic features of more than one lesion, referred to as "hybrid" lesions. ${ }^{11}$ CPAMs are usually limited to one lobe. ${ }^{12}$ Hence, prenatal ultrasound of a microcystic CPAM often demonstrates an echogenic solid appearing lesion neighboring normal ipsilateral lung tissue. However, our preoperative images demonstrated an unconventional appearance of the microcystic CPAM; the entire right thorax was filled with an echogenic mass. The overexpanded echogenic right lung was remarkably similar to prenatal ultrasound images of a congenital diaphragmatic hernia $(\mathrm{CDH})$ treated with fetoscopic endotracheal occlusion ${ }^{13-16}$ or a CHAOS. ${ }^{17}$ However, in the setting of CDH or CHAOS, both lungs appear symmetrically enlarged and echogenic due to an obstruction at the level of the trachea or more superiorly. Given the unusual presentation of our patient, fetal bronchoscopy allowed us to exclude the presence of a bronchial lesion from the differential diagnosis. The $1.1 \mathrm{~cm}$ cystic structure within the lung parenchyma did not provide an explanation for the enlarged right lung due to its relative small size and absence of dilated distal bronchi. Had an obstructive bronchial lesion been identified, laser perforation could have removed the obstruction and allowed efflux of the trapped fluid. Thus, fetal bronchoscopy provided important diagnostic information in this case.

With a bronchial obstruction ruled out, the leading diagnosis was a microcystic CPAM, which could be treated in utero via maternal steroid administration, ${ }^{3}$ open fetal resection, ${ }^{2}$ or percutaneous sclerotherapy. ${ }^{5,18}$ However, this was not necessary because the CPAM significantly reduced in size after the fetal bronchoscopy. There are three potential reasons why the CPAM reduced in size. First, the natural history of CPAMs is such that reduction in size in the third trimester is common. ${ }^{19}$ Second, the two courses of steroids given in this case may have facilitated the reduction in tumor size. ${ }^{7,8}$ The mean (one standard deviation) time for resolution of hydrops after multiple betamethasone courses for congenital lung lesions was previously reported as $33 \pm 32$ days. ${ }^{8}$ Hence, it remains plausible that continued expectant management after the two courses of betamethasone could have still ultimately led to the decrease in lung mass size and resolution of hydrops. A third possible reason for the apparent reduction in tumor size post procedure may be due to the procedure itself. We hypothesize that the saline used to aide 
in visualization inadvertently dislodged debris that was, in part, obstructing the lower airways. Perhaps the origin of this debris was mucoid material produced by the aggregates of mucus producing cells within the CPAM, as was noted in the histopathological specimens (-Fig. 4). We speculate that prior to the lavage, the mucus produced by the CPAM located in the right upper lobe was disseminated diffusely and obstructing multiple bronchi in all three lobes of the right lung. Hence, the lavage may have dislodged and washed out enough of the mucus to restore the natural efflux of pulmonary secretions over time, leaving only the focal echogenic CPAM seen on postoperative ultrasounds and postnatal $\mathrm{CT}$. In other words, we posit that the saline lavage may have had an inadvertent therapeutic benefit in this case. This assertion is purely conjecture at this time based on the fetoscopic visualization of mucoid material at the time of saline lavage and the pathological confirmation of mucus producing cells in the specimen. Furthermore, we acknowledge the possibility that the mucoid material is a normal component of fetal lung development and may be present in a great majority of normal fetuses.

Mucus producing cells are seen in approximately 35\% of CPAM Type I cases but traditionally not associated with CPAM Type II. ${ }^{20}$ However, there is a case report of an adult patient with recurrent pneumonia that underwent a left lower lobectomy for a CPAM Type II. There was no mention of diffuse mucoid material as seen in our case, but a $1.5 \times 1.5 \mathrm{~cm}^{2}$ cyst did contain inspissated mucus. ${ }^{21}$ Perhaps the subset of children that are expectantly managed with CPAM and later develop pulmonary infections, do in part, because of a build-up of mucoid secretions.

Quintero et al described a similar case in which a fetus with a large left hyperechogenic lung mass and a small hypoechoic cystic structure near the hilum underwent fetal bronchoscopy at $31^{5 / 7}$ weeks. ${ }^{9}$ Preoperatively, the fetus had no normal lung tissue on the affected left side and a compressed contralateral right lung. The differential diagnosis included CPAM Type III, pulmonary sequestration, bronchogenic cyst or lobar bronchial atresia. During the fetal bronchoscopy, normal left lung tissue became delineated and there was expansion of the right lung. Following the bronchoscopy, the left hyperechogenic mass significantly regressed and the right lung expanded prior to delivery. The saline lavage utilized during bronchoscopy was believed to have increased airway pressure and established luminal patency in bronchi that may have been occluded with inspissated material. Postnatally, surgical pathology was consistent with a hybrid lesion of CPAM Type III and extralobar sequestration. $^{9}$

The case by Quintero et $\mathrm{al}^{9}$ and the current case further highlight the challenges in classifying CPAMs. ${ }^{22,23}$ Perhaps these cases represent a rare form of CPAM in which a transient diffuse bronchial obstruction is caused by excessive mucus production. This theory offers an explanation for the antenatal ultrasound appearance of the microcystic appearance of the enlargement of the entire right lung, and the potential therapeutic effect the bronchial lavage had in restoring normal lung size and appearance. However, it remains unclear if the mucin inspissation was the underlying pathology leading to subsequent abnormal bronchial development, or it was an incidental finding in a primary developmental malformation of the lower respiratory tract.

Fetal bronchoscopy has advantages over neonatal bronchoscopy. First, fetal bronchoscopy is performed while on placental circulation and therefore does not have the same risks of deoxygenation as in a neonatal bronchoscopy. Second, direct visualization narrows the differential diagnosis. As previously discussed, the degree of hyperechogenicity and diaphragm eversion in our case was not consistent with the typical findings of a microcystic CPAM. If an obstruction, such as bronchial atresia is encountered in utero, laser energy can be used to perforate the obstruction and reestablish luminal patency. ${ }^{6,24}$ This not only allows the stored pulmonary mucus secretions to be expelled, but also normalization of the fetal lung tissue, correction of the mediastinal shift, and resolution of the hydrops fetalis. ${ }^{6,24}$

The advantages of fetal bronchoscopy need to be weighed against its limitations. First, the risk of PPROM and preterm delivery should be considered when determining the timing of the intervention. Such risks should not be underestimated, particularly when prematurity would compound the morbidity to an already at risk fetus. To mitigate complications, an invasive procedure as rare as fetal bronchoscopy should be performed only by an experienced team of providers. Second, fetal position may not be optimal for bronchoscopy and external manipulation may be required. Third, fetal bronchoscopy should be reserved for obstructive lower airway diseases that potentially can be improved by re-establishing bronchial patency. Alternative management strategies need to be considered carefully, and fetal bronchoscopy should be attempted only if the alternatives are deemed inadequate treatment. In our case, performing only an amnioreduction without a fetal bronchoscopy would potentially have prolonged the gestation without correcting the risk of pulmonary hypoplasia. Given the paucity of literature on fetal bronchoscopy, it remains unclear to what extent, if any, the saline irrigation used during bronchoscopy is helpful in clearing obstructive debris.

In summary, the collective evidence from previously published fetal tracheobronchoscopy cases ${ }^{6,9,24,25}$ and ours adds to the current understanding of prenatal diagnosis and management of congenital lung lesions. Under the proper circumstances, fetal tracheobronchoscopy can serve as a diagnostic tool, as well as a possible therapeutic measure if an obstructive lesion is identified. The role of fetal bronchial lavage in the rare instances of a mucoid producing CPAM requires further investigation at specialized fetal therapy centers.

\section{Conflict of Interest}

The authors report no conflict of interest.

\section{References}

1 Morris LM, Lim FY, Livingston JC, Polzin WJ, Crombleholme TM. High-risk fetal congenital pulmonary airway malformations have a variable response to steroids. J PediatrSurg 2009;44(01):60-65

2 Loh KC, Jelin E, Hirose S, Feldstein V, Goldstein R, Lee H. Microcystic congenital pulmonary airway malformation with hydrops 
fetalis: steroids vs open fetal resection. J PediatrSurg 2012;47 (01):36-39

3 Curran PF, Jelin EB, Rand L, et al. Prenatal steroids for microcystic congenital cystic adenomatoid malformations. J PediatrSurg 2010; 45(01):145-150

4 Quintero RA, Quintero LF, Chmait R, et al. The quantitative lung index (QLI): a gestational age-independent sonographic predictor of fetal lung growth. Am J ObstetGynecol 2011;205(06):544.e1-544.e8

5 Lee FL, Said N, Grikscheit TC, Shin CE, Llanes A, Chmait RH. Treatment of congenital pulmonary airway malformation induced hydrops fetalis via percutaneous sclerotherapy. Fetal DiagnTher 2012;31(04):264-268

6 Martínez JM, Prat J, Gómez O, et al. Decompression through tracheobronchial endoscopy of bronchial atresia presenting as massive pulmonary tumor: a new indication for fetoscopic surgery. Fetal DiagnTher 2013;33(01):69-74

7 Derderian SC, Coleman AM, Jeanty C, et al. Favorable outcomes in high-risk congenital pulmonary airway malformations treated with multiple courses of maternal betamethasone. J PediatrSurg 2015;50(04):515-518

8 Peranteau WH, Boelig MM, Khalek N, et al. Effect of single and multiple courses of maternal betamethasone on prenatal congenital lung lesion growth and fetal survival. J PediatrSurg 2016; 51(01):28-32

9 Quintero RA, Kontopoulos E, Reiter J, Pedreira WL, Colin AA. Fetal bronchoscopy: its successful use in a case of extralobar pulmonary sequestration. J Matern Fetal Neonatal Med 2012;25(11):2354-2358

10 Ankermann T, Oppermann HC, Engler S, Leuschner I, Von Kaisenberg CS. Congenital masses of the lung, cystic adenomatoid malformation versus congenital lobar emphysema: prenatal diagnosis and implications for postnatal treatment. J Ultrasound Med 2004;23(10):1379-1384

11 Winters WD, Effmann EL. Congenital masses of the lung: prenatal and postnatal imaging evaluation. J Thorac Imaging 2001;16(04): 196-206

12 Evrard V, Ceulemans J, Coosemans W, et al. Congenital parenchymatous malformations of the lung. World J Surg 1999;23(11): $1123-1132$

13 Jordan EJ, Feldstein VA, Moses W, Phelps AS. Radiologic assessment of fetal tracheal balloon occlusion. Clin Imaging 2017;42:113-118
14 Claus F, Sandaite I, DeKoninck P, et al. Prenatal anatomical imaging in fetuses with congenital diaphragmatic hernia. Fetal DiagnTher 2011;29(01):88-100

15 Kohl T, Gembruch U, Filsinger B, et al; German Center for Fetal Surgery Diaphragmatic Hernia Task Group. Encouraging early clinical experience with deliberately delayed temporary fetoscopic tracheal occlusion for the prenatal treatment of life-threatening right and left congenital diaphragmatic hernias. Fetal DiagnTher 2006;21(03):314-318

16 Ruano R, Ali RA, Patel P, Cass D, Olutoye O, Belfort MA. Fetal endoscopic tracheal occlusion for congenital diaphragmatic hernia: indications, outcomes, and future directions. ObstetGynecolSurv 2014;69(03):147-158

17 Resnik R, Creasy RK, Iams JD, Lockwood CJ, Moore T, Greene FM. Creasy and Resnik's Maternal-Fetal Medicine: Principles and Practice. 7th ed. Saunders/Elsevier; 2013

18 Bermúdez C, Pérez-Wulff J, Arcadipane M, et al. Percutaneous fetal sclerotherapy for congenital cystic adenomatoid malformation of the lung. Fetal DiagnTher 2008;24(03):237-240

19 Cavoretto P, Molina F, Poggi S, Davenport M, Nicolaides KH. Prenatal diagnosis and outcome of echogenic fetal lung lesions. Ultrasound ObstetGynecol 2008;32(06):769-783

20 Stocker JT. Cystic lung disease in infants and children. Fetal PediatrPathol 2009;28(04):155-184

21 Baral D, Adhikari B, Zaccarini D, Dongol RM, Sah B. Congenital pulmonary airway malformation in an adult male: a case report with literature review. Case Rep Pulmonol 2015;2015:743452

22 Fowler DJ, Gould SJ. The pathology of congenital lung lesions. SeminPediatrSurg 2015;24(04):176-182

23 Kotecha S, Barbato A, Bush A, et al. Antenatal and postnatal management of congenital cystic adenomatoid malformation. PaediatrRespir Rev 2012;13(03):162-170, quiz 170-171

24 Cruz-Martinez R, Méndez A, Perez-Garcilita O, et al. Fetal bronchoscopy as a useful procedure in a case with prenatal diagnosis of congenital microcysticadenomatoid malformation. Fetal DiagnTher 2015;37(01):75-80

25 Quintero R, Hale-Burnett E, Bornick PW, Gilbert-Barness E. Fetal laryngoscopy and lung biopsy in a case of bilateral lethal congenital cystic adenomatoid malformation of the lung. Fetal PediatrPathol 2007;26(5-6):229-234 